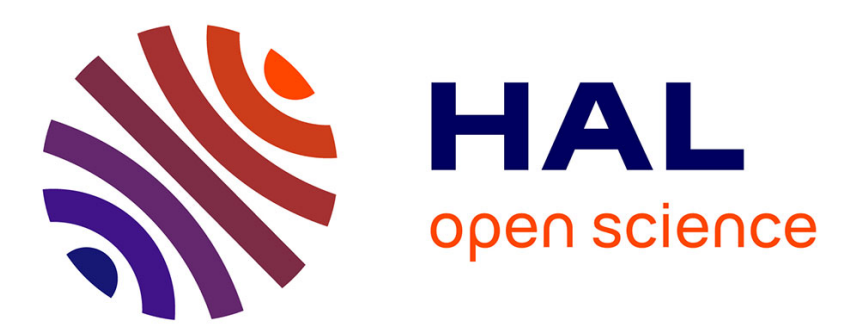

\title{
Calibration of galvanometric scan heads for additive manufacturing with machine assembly defects consideration
}

Kevin Godineau, Sylvain Lavernhe, Christophe Tournier

\section{- To cite this version:}

Kevin Godineau, Sylvain Lavernhe, Christophe Tournier. Calibration of galvanometric scan heads for additive manufacturing with machine assembly defects consideration. Additive Manufacturing, 2019, 26, pp.250-257. 10.1016/j.addma.2019.02.003 . hal-02011888

\section{HAL Id: hal-02011888 \\ https://hal.science/hal-02011888}

Submitted on 8 Feb 2019

HAL is a multi-disciplinary open access archive for the deposit and dissemination of scientific research documents, whether they are published or not. The documents may come from teaching and research institutions in France or abroad, or from public or private research centers.
L'archive ouverte pluridisciplinaire $\mathbf{H A L}$, est destinée au dépôt et à la diffusion de documents scientifiques de niveau recherche, publiés ou non, émanant des établissements d'enseignement et de recherche français ou étrangers, des laboratoires publics ou privés. 


\title{
Calibration of galvanometric scan heads for additive manufacturing with machine assembly defects consideration
}

\author{
K. Godineau ${ }^{\mathrm{a}}$, S. Lavernhe ${ }^{\mathrm{a}}$, C. Tournier ${ }^{\mathrm{a}}$ \\ ${ }^{a}$ LURPA, ENS Paris-Saclay, Institut Farman (FR3311), Univ. Paris-Sud, Université Paris-Saclay, 94235 Cachan, France
}

\begin{abstract}
The calibration of additive manufacturing machines using scanning heads in processes such as Laser Powder Bed Fusion (LPBF) and vat photopolymerization is an iterative and time-consuming process often based on limited physical models. Indeed, the relationship between the laser spot position in the work plane and the actuators position (galvanometers) is achieved by interpolating correction tables experimentally determined. In this paper, representative geometrical models of the real system are established in order to reduce the time required to obtain the final correction tables. For this purpose, a geometrical model is developed with assembly defects consideration. This model is used in a process of defects identification to obtain a virtual machine representative of the real system and thus directly generates the final correction tables. The geometrical model thereby developed is used to quantify the impact of assembly defects on the laser spot position, to compensate them and to reduce the calibration time of an additive manufacturing machine.
\end{abstract}

Keywords:

Additive manufacturing, Assembly defects, Geometrical model, Virtual machine, Calibration

\section{Introduction}

Laser Powder Bed Fusion (LPBF) and vat photopolymerization processes use a laser beam to fuse the material (powder or polymer) in order to build a part layer by layer. The geometrical dimensions and mechanical characteristics of the produced parts using these three processes mainly depend on the control of the laser spot position and the energy transferred to the material. The laser spot position is controlled by a laser scanning system also called a "galvanometric head". The movement of the laser spot is obtained by deflecting the laser beam through two rotary mirrors actuated by galvanometers (Fig. 1). Laser scanning devices are made of a first device focusing the laser beam in the work plane and a second one for positioning the laser spot in the work plane. There are mainly two types of focusing systems [1], one called f-theta lens located after the positioning device (pre-objective scan system), and the other one called dynamic focus module (DFM) located before the positioning device (post-objective scan system).

In order to generate the set points sent to the galvanometers, it is necessary to generate a relationship between the laser spot position in the work plane $\boldsymbol{X}$ (task space) and the position of the different actuators $\boldsymbol{Q}$ (joint space). In additive manufacturing, this relationship is achieved by using correction tables (also call lookup tables). The principle of these tables is to construct a grid of points in the task space and to associate at each node of the grid the corresponding joint position [2-4]. There is one correction table for each joint component. Once the tables

\footnotetext{
Email address: kevin.godineau@ens-paris-saclay.fr (K. Godineau )
}

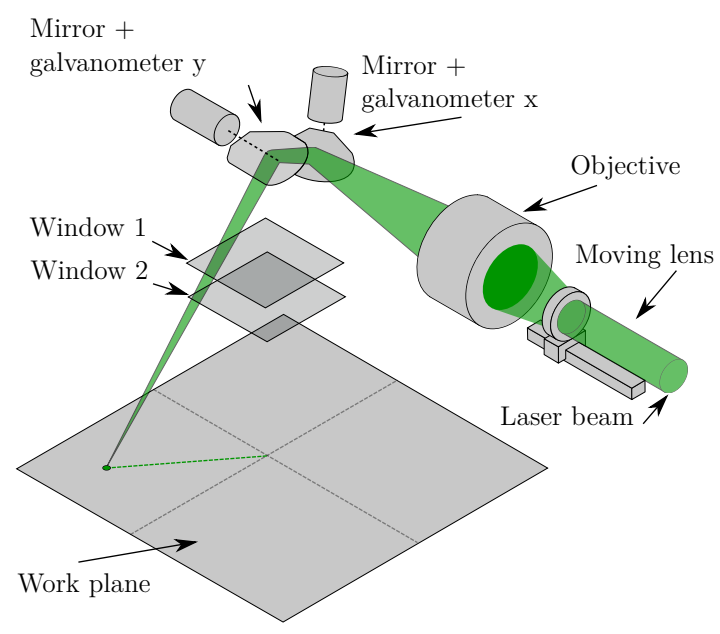

Figure 1: Opto-mechanical chain of an additive manufacturing machine with dynamic focus module (DFM) - RAYLASE

are generated, it is possible to determine the joint instructions associated with a position in the task space by the interpolation of grids. The errors introduced by the correction tables are directly related to the interpolation strategy (bilinear, bicubic, etc.) and to the tables resolution (number of tabulated values). This method allows to obtain a simple mathematical relationship between the task space and the joint space which is essential considering the $100 \mathrm{kHz}$ instructions frequency required to control the system.

The generation of correction tables is a tiresome process because it requires many experimental measurements. Indeed, it is necessary to conduct real shots and measurements to recali- 
brate the system. This process is repeated until a suitable marking is obtained. To overcome this issue, many works [3, 5-7] identify the behavior of the laser scanning systems by a polynomial with two variables (position coordinates $x$ and $y$ of the laser spot in the work plane). The identification of the coefficients is performed by approximating or interpolating experimental data. This global approach requires few measurement points (or marker) which reduces the calibration time but does not allow to identify local behaviours and depends strongly on experimental data properties (number of points, measurement noise, position of the points). More local approaches have therefore been developed to generate correction tables. For example, the work described in [4] presents a hardware architecture that measures the local position of the laser spot by inserting a camera into the optical chain. The laser spot position is then determined with respect to a reference grid. Local or global calibration methods do not integrate the geometry of the scanning system and are only based on experimental measurements.

Several mathematical models have been established to take geometry into account when calibrating laser scanning systems $[2,3,8]$. These models provide a good approximation of the actual behaviour of the systems. However, these models are based on restrictive geometrical hypotheses: mirrors rotary axes confused with their reflection surfaces, simplified rotary axes orientation...

More representative models have therefore been developed to consider different sources of defects, study their impacts on the laser spot position in the work plane and compensate them. The works [6,9-11] describe numerous sources of defects (dynamical [12, 13], geometrical, thermal [14] and optical) relating to the galvanometer + mirror systems. Geometrical defects (assembly defects and shape of the lens) have the highest influence on the laser spot position. The works [8] and [15] focus on the position and orientation defects of the laser source whereas [6] and [4] focus on the work plane defects relative to the laser scanning head. The work done in [16] provides a model with 13 parameters and study the influence of each parameter on the laser spot position. However, these parameters only represent defects related to the laser source orientation and to the galvanometric head (positions and orientations of the mirrors and their axes).

All these defects sources make it difficult to obtain a complete analytical model. That's why calibration protocols use simple geometric models to pre-calibrate laser scanning systems. The work presented in this paper provides a continuous and accurate geometrical model of the opto-mechanical chain (laser source, galvanometric head, windows, work plane) which can be directly used in a calibration protocol to obtain the final correction tables in a single measurement step.

The main claims of this article are:

1. A nominal geometrical model of the laser scanning system without restrictive assumptions about the optical chain geometry.

2. A geometrical model with 30 assembly defects taken into account.
3. A hierarchy of the impact of geometrical assembly defects on the laser spot position.

4. A method of identifying assembly defects to calibrate additive manufacturing machines via a single measurement phase.

The rest of the paper is organized as follows. The nominal model is introduced in the second section and is completed by the consideration of assembly defects in the third section. An analysis of each defect impact on the laser spot position in the work plane is then performed. Section 4 is dedicated to the identification of the 30 defects of the model by using data from experiments on an industrial LPBF machine. Tests are then conducted with the new identified correction tables to evaluate the gains obtained on the real machine. Finally, the paper is concluded in section 5 .

\section{Nominal Model}

The opto-mechanical chain studied is composed of a laser source, two rotary mirrors reflecting the laser beam, two windows to separate the production environment and the laser source environment, and a work plane in which the material is fused (Fig. 1 and Fig. 2).

\subsection{Assumptions and parameterization}

To describe the laser beam path, the points $P_{i}$ and the vectors $\vec{u}_{i}$ are introduced. The points $P_{i}$ represent the intersection of the laser beam with the different surfaces of the optical chain elements (Fig. 1). The vectors $\vec{u}_{i}$ represent the unit vectors of the same direction as the vectors $\overrightarrow{P_{i} P_{i+1}}$ (Eq. (1)).

$$
\overrightarrow{u_{i}}=\frac{\overrightarrow{P_{i} P_{i+1}}}{\left\|\overrightarrow{P_{i} P_{i+1}}\right\|}
$$

The following assumptions are made to describe the nominal geometry of the system:

1. The intersection between the laser beam and the work plane is not influenced by the focusing device.

2. Laser source origin $O_{s}$ is located in the center of the equivalent lens (of variable focal length) of the focusing device.

3. The laser beam intersects the work plane perpendicularly in the following joint configuration $\left(\theta_{x}, \theta_{y}\right)=(0,0)$. The intersection point is named $O_{p}$ (origin of the coordinate system linked to the work plane).

4. The surfaces of the mirrors, work plane, and windows are considered perfectly flat.

5. The thickness of the windows is constant and equal to $e_{v}$; the window surfaces are therefore parallel.

6. Windows surfaces are parallel to the surface of the work plane.

7. Assumptions of geometrical optics are considered, therefore the laser beam is modeled by a light beam.

8. The distance between the mirror rotary axis and the mirror reflection surface is non-zero and is named $e_{m}$. 


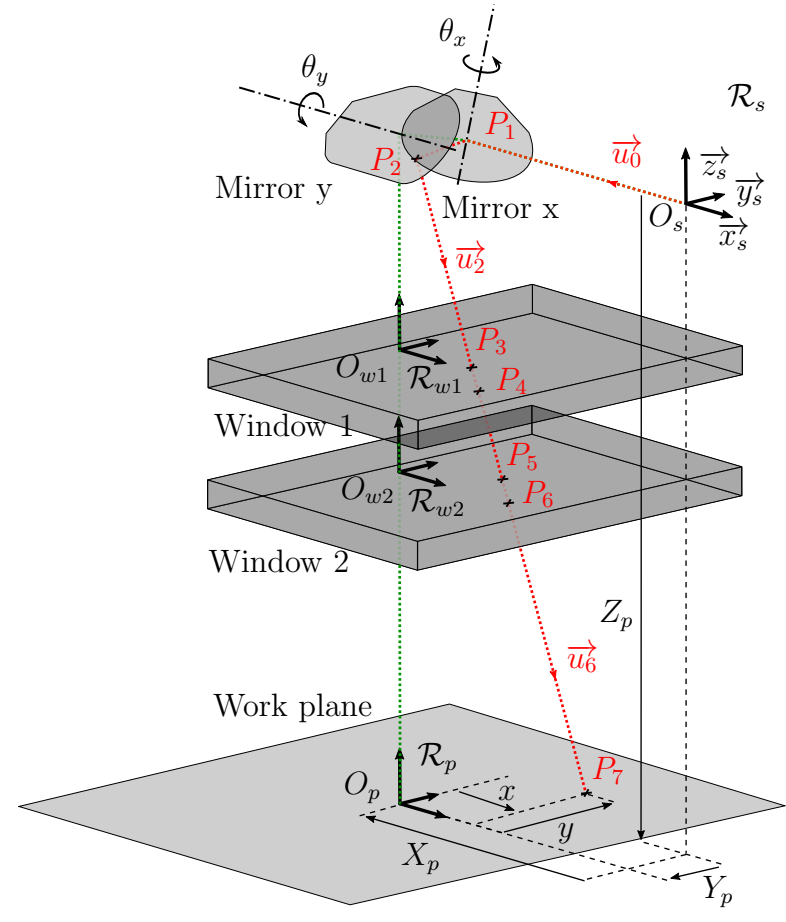

Figure 2: Optical chain parameterization of an additive manufacturing machine - the green laser beam corresponds to the joint configuration $\left(\theta_{x}, \theta_{y}\right)=(0,0)$

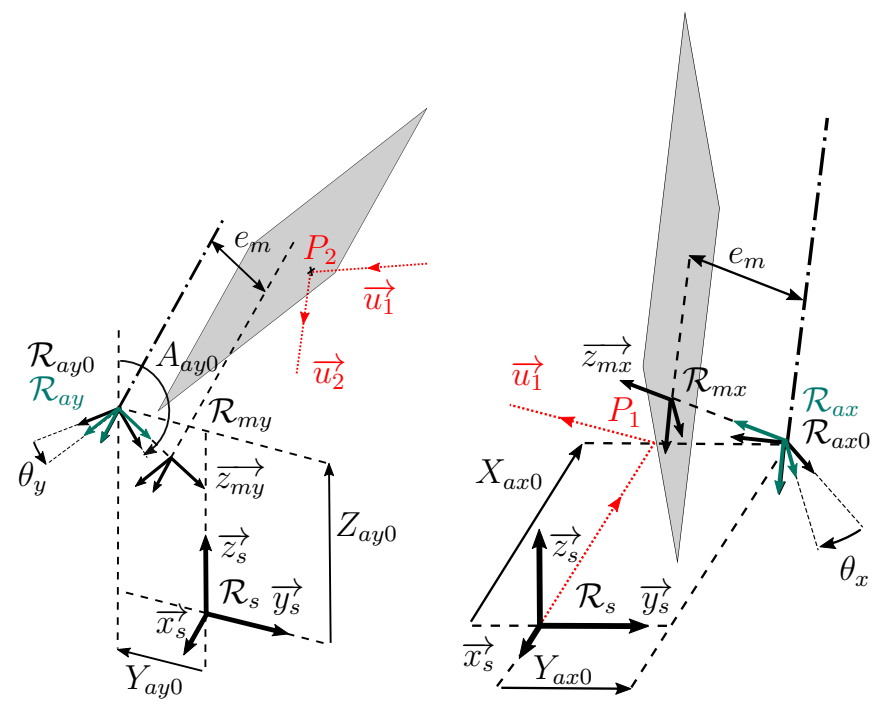

Figure 3: Mirrors parameterization details of the Fig. 2

\subsection{Computation of the forward kinematic model}

The forward kinematic model (FKM) expresses the coordinates of the laser spot position in the work plane $\boldsymbol{X}=(x, y)=$ $\overrightarrow{O_{p} P_{7}}$ as a function of the joint commands $\boldsymbol{Q}=\left(\theta_{x}, \theta_{y}\right)$. This model represents a nominal virtual machine and thus makes it possible to simulate the behavior of an additive manufacturing machine.

The forward kinematic model is calculated by following the optical path. In this way, the laser spot position $P_{7}$ is expressed in the work plane coordinate system $\mathcal{R}_{p}$ and is decomposed into a vectorial sum (Eq. (2)).

$$
\overrightarrow{O_{p} P_{7}}=\overrightarrow{O_{p} O_{s}}+\overrightarrow{O_{s} P_{1}}+\overrightarrow{P_{1} P_{2}}+\ldots+\overrightarrow{P_{6} P_{7}}
$$

The first vector $\overrightarrow{O_{p} O_{s}}$ is known and defined by the geometrical parameters $X_{p}, Y_{p}$ and $Z_{p}$. The second vector $\overrightarrow{O_{s} P_{1}}$ (laser direction) defines the unit vector $\vec{x}_{s}$. The point $P_{1}$ is obtained by projecting $O_{s}$ on the mirror surface $x$ in the direction $\overrightarrow{u_{0}}=-\overrightarrow{x_{s}}$. By knowing the normal of the mirror surface $\overrightarrow{z_{m x}}$ and any point on the mirror $O_{m x}$ it is possible to determine the vector $\overrightarrow{O_{s} P_{1}}$ (Eq. (3)):

$$
\overrightarrow{O_{s} P_{1}}=\left(\frac{\overrightarrow{O_{s} O_{m x}} \cdot \overrightarrow{z_{m x}}}{\overrightarrow{x_{s}} \cdot \overrightarrow{z_{m x}}}\right) \overrightarrow{x_{s}}
$$

The vector $\overrightarrow{u_{1}}$ corresponds to the reflection of the vector $\overrightarrow{u_{0}}$ on the mirror $x$ (Eq. (4)):

$$
\overrightarrow{u_{1}}=\overrightarrow{u_{0}}-2\left(\overrightarrow{z_{m x}} \cdot \overrightarrow{u_{0}}\right) \cdot \overrightarrow{z_{m x}}
$$

The vector $\overrightarrow{u_{2}}$ is computed in the same manner as the vector $\overrightarrow{u_{1}}$. The computation of $\overrightarrow{P_{1} P_{2}}\left(\overrightarrow{P_{2} P_{3}}\right)$ is achieved by projecting the point $P_{1}\left(P_{2}\right)$ on the mirror $y$ (on window $w_{1}$ ) in the direction $\overrightarrow{u_{1}}\left(\overrightarrow{u_{2}}\right)$. This computation is similar to the computation of the vector $\overrightarrow{O_{s} P_{1}}$. Vectors $\overrightarrow{P_{3} P_{4}}$ and $\overrightarrow{u_{4}}$ are expressed using the laws of geometrical optics. Two optical parameters are introduced here, the refractive index of the external environment $n_{1}$ and the refractive index of the windows $n_{2}$.

$$
\overrightarrow{P_{3} P_{4}}= \begin{cases}-e_{v}\left(\overrightarrow{z_{w 1}}-\frac{D}{\sqrt{1-D^{2}}} \times \overrightarrow{t_{w 1}}\right) & \text { if } \overrightarrow{u_{2}} \wedge \overrightarrow{z_{w 1}} \neq \overrightarrow{0} \\ -e_{v} \times \overrightarrow{z_{w 1}} & \text { if } \overrightarrow{u_{2}} \wedge \overrightarrow{z_{w 1}}=\overrightarrow{0}\end{cases}
$$

with $\overrightarrow{t_{w 1}}=\frac{\overrightarrow{z_{w 1}} \wedge\left(\overrightarrow{u_{2}} \wedge \overrightarrow{z_{w 1}}\right)}{\left\|\overrightarrow{u_{2}} \wedge \overrightarrow{z_{w 1}}\right\|}$ and $\quad D=\frac{n_{1}}{n_{2}} \sqrt{1-\left(-\overrightarrow{u_{2}} \cdot \overrightarrow{z_{w 1}}\right)^{2}}$

$$
\overrightarrow{u_{4}}=\overrightarrow{u_{2}}
$$

Vectors $\overrightarrow{P_{4} P_{5}}, \overrightarrow{P_{5} P_{6}}$ and $\vec{u}_{6}$ are obtained in the same way as vectors $\overrightarrow{P_{2} P_{3}}, \overrightarrow{P_{3} P_{4}}$ and $\overrightarrow{u_{4}}$. Finally, the vector $\overrightarrow{P_{6} P_{7}}$ is obtained by projecting the point $P_{6}$ (previously calculated) on the work plane in the direction $\overrightarrow{u_{6}}$.

In order to obtain an analytical formulation of Eq. (2), it is necessary to define all vectors $\left(x_{s}, \overrightarrow{z_{m x}}, \overrightarrow{z_{m y}}, \overrightarrow{z_{w 1}}, \overrightarrow{z_{w 2}}, \overrightarrow{z_{p}}\right)$ and all points used $\left(O_{s}, O_{m x}, O_{m y}, O_{w 1}, O_{w 2}, O_{p}\right)$ in the same coordinate system. By expressing all these elements in the same coordinate system, the control parameters $\theta_{x}$ and $\theta_{y}$ as well as all the geometric parameters appear. The change of coordinate systems is performed using homogeneous transformation matrices defined as follows: ${ }^{j} \boldsymbol{T}_{i}$ is the transformation matrix from the coordinate system $\mathcal{R}_{i}$ to the coordinate system $\mathcal{R}_{j}$, and $\boldsymbol{R}$ the associated rotation matrix described by an extrinsic Tait Bryan parameterization.

$$
{ }^{j} \boldsymbol{T}_{i}=\left(\begin{array}{rrrr} 
& & & X \\
& \boldsymbol{R} & Y \\
& & & Z \\
0 & 0 & 0 & 1
\end{array}\right) \text { with } \quad \boldsymbol{R}=\boldsymbol{R}_{z}(C) \cdot \boldsymbol{R}_{y}(B) \cdot \boldsymbol{R}_{x}(A)
$$


The following notation is used to simplify matrix writing: ${ }^{j} \boldsymbol{T}_{i}=$ $(X, Y, Z, A, B, C)$. All the transformation matrices required to develop the nominal geometrical model are described in the following list:

$$
\begin{aligned}
& \text { - }{ }^{\text {plane }} \boldsymbol{T}_{\text {source }}=\left(X_{p}, Y_{p}, Z_{p}, 0,0,0\right) \\
& \text { - }{ }^{\text {axis_x } 0} \boldsymbol{T}_{\text {source }}=\left(X_{a x 0}, Y_{a x 0}, 0, A_{a x 0}, B_{a x 0}, 0\right) \\
& \text { - }{ }^{\text {axis_x } x} \boldsymbol{T}_{\text {axis_x } 0}=\left(0,0,0, \theta_{x}, 0,0\right) \\
& \text { - }{ }^{\text {mirror_x } x} \boldsymbol{T}_{\text {axis_x }}=\left(0,0, e_{m}, 0,0,0\right) \\
& \text { - }{ }^{\text {axis_y } 0} \boldsymbol{T}_{\text {source }}=\left(0, Y_{a y 0}, Z_{a y 0}, A_{a y 0}, 0,0\right) \\
& \text { - }{ }^{\text {axis_y } y} \boldsymbol{T}_{\text {axis_y } 0}=\left(0,0,0, \theta_{y}, 0,0\right) \\
& \text { - }{ }^{\text {mirror-y } y} \boldsymbol{T}_{\text {axis_y }}=\left(0,0, e_{m}, 0,0,0\right)
\end{aligned}
$$

Considering previous assumptions, the simplification of Eq. (2) removes the positioning parameters of the windows. Therefore, for windows, only orientation has an influence on the laser spot position. According to the hypothesis $n^{0} 6$ it is possible to replace the vectors $\overrightarrow{z_{w 1}}$ and $\overrightarrow{z_{w 2}}$ by $\overrightarrow{z_{p}}$. Once this last step is completed, the FKM is obtained.

\subsection{Forward kinematic model}

The resulting FKM obtained from the simplification of Eq. (2) is detailed in Eqs. (8), (9). In order to decrease the size of the equations, the variables $K_{1}, K_{2}$ and $K_{3}$ are introduced (Eqs. (10), (11), (12)).

$$
\begin{gathered}
K_{1}=\frac{e_{m}+Z_{a y 0} \cos \left(A_{a y 0}+\theta_{y}\right)-Y_{a y 0} \sin \left(A_{a y 0}+\theta_{y}\right)}{\cos \left(A_{a y 0}-B_{a x 0}+\theta_{y}\right)} \\
K_{2}=Z_{p}+2 e_{v}-K_{1} \cos \left(B_{a x 0}\right) \\
K_{3}=\sqrt{\cos \left(2 \theta_{x}\right)^{2} \cos \left(2 \theta_{y}\right)^{2}-1+\frac{n_{2}^{2}}{n_{1}^{2}}}
\end{gathered}
$$

Moreover, to simplify the notations, in the rest of the paper the FKM is represented by the function $h$, which allows to rewrite the Eqs. (8) and (9) under the following form:

$$
\boldsymbol{X}=h(\boldsymbol{Q})
$$

Considering the following values: $e_{v}=0, e_{m}=0, X_{a x 0}=$ $0, Y_{a x 0}=0, Z_{a y 0}=0, A_{a x 0}=-45^{\circ}, B_{a x 0}=90^{\circ}, A_{a y 0}=-135^{\circ}$ it is possible to retrieve the models developed in the work [2], [3] and [8] where the only remaining parameters are $Y_{a y 0}, Z_{p}$, $\theta_{x}$ and $\theta_{y}$. The nominal model developed in this article helps to understand the impact of the various geometrical parameters on the laser spot position in the case of a post-lens system. For example, the integration of the thickness $e_{m}$ between the mirrors rotary axis and their reflection surfaces allow to describe the variation of the point position $P_{2}$ on the mirror surface. Due to its low mathematical complexity, this geometrical model can be used directly in real-time control/command applications.

\subsection{Inverse kinematic model}

In order to determine the joint commands to be sent to the actuators to achieve the laser spot path, it is necessary to compute the inverse kinematic model (IKM). This model is defined by Eq. (14).

$$
\boldsymbol{Q}=h^{-1}(\boldsymbol{X})
$$

The inverse kinematic model is obtained by isolating $\theta_{x}$ and $\theta_{y}$ in the system formed by Eqs. (8) and (9). This system cannot be inverted analytically, therefore the IKM is solved numerically by a Newton-Raphson method. The Jacobian matrix is pre-calculated in order to minimize the computation time. However, the iterative resolution of the IKM to perform realtime computations at a frequency of $100 \mathrm{kHz}$ is not achievable. It is therefore necessary to discretize the IKM into correction tables in order to be able to perform real time calculations in the numerical controller.

The positioning accuracy of the laser spot depends essentially on the correlation between the IKM and the actual geometry of the machine. Moreover, the IKM is obtained from the FKM, thus the calibration step of an additive manufacturing machine consists in the determination of a FKM as representative as possible of the real machine.

\section{Model with assembly defects consideration}

In order to obtain a reliable FKM of the opto-mechanical chain of the real system, it is necessary to enhance the previous nominal model by introducing behaviours inherent to each machine such as assembly defects, optical defects, etc. The work developed in this article deals more specifically with modelling and integrating the assembly defects of the various components (laser source, mirrors axes, mirrors surfaces, windows, work plane) in the previous model.

\subsection{Assembly defects modelling}

Assembly defects consist in displacement and reorientation of each component compared with their nominal position. A position's defect of the element $i$ along the axis $x$ ( $y$ or $z$ ) is noted $\delta x_{i}$ (respectively $\delta y_{i}$ and $\delta z_{i}$ ). Orientation defects around $x, y$ and $z$ axes are denoted respectively $\delta a_{i}, \delta b_{i}$ and $\delta c_{i}$.

The 6 components of assembly defects do not necessarily have an impact on the laser spot position in the work plane. For example, the laser source is oriented along the $\vec{x}_{s}$ axis, so it is not sensitive to a misalignment around $\overrightarrow{x_{s}}\left(\delta a_{s}\right)$, and to a positioning error along $\vec{x}_{s}\left(\delta x_{s}\right)$. For each element of the optomechanical chain, the list of all components having an impact on the laser position is summarized in Table 1.

To implement these geometrical defects in the nominal model, new coordinate systems with $d$ subscripts are defined and associated to each element in their actual situations. The transformation matrices between nominal situations and the actual ones are listed as follows:

$$
\begin{aligned}
& \text { - }{ }^{\text {source }} \boldsymbol{T}_{\text {source_d }}=\left(0, \delta y_{s}, \delta z_{s}, 0, \delta b_{s}, \delta c_{s}\right) \\
& \text { - }{ }^{\text {axis_x } x 0_{-} d} \boldsymbol{T}_{\text {axis_x } 0}=\left(0, \delta y_{a x}, \delta z_{a x}, \delta a_{a x}, \delta b_{a x}, \delta c_{a x}\right)
\end{aligned}
$$




$$
\begin{gathered}
x=-X_{p}+X_{a x 0}+\frac{e_{m}-Y_{a x 0} \cos \left(-A_{a x 0}+\theta_{x}\right) \sin \left(B_{a x 0}\right)}{\sin \left(-A_{a x 0}+\theta_{x}\right)}-K_{2} \frac{\tan \left(2 \theta_{x}\right)}{\cos \left(2 \theta_{y}\right)}-K_{1} \tan \left(2 \theta_{x}\right)+\frac{2 e_{v} \sin \left(2 \theta_{x}\right)}{K_{3}} \\
y=-Y_{p}-K_{1} \sin \left(B_{a x 0}\right)-K_{2} \tan \left(2 \theta_{y}\right)+\frac{2 e_{v} \cos \left(2 \theta_{x}\right) \sin \left(2 \theta_{y}\right)}{K_{3}}
\end{gathered}
$$

Table 1: List of assembly defects that affect the laser spot position in the work plane coordinate system.

\begin{tabular}{l|llllll} 
& $\mathrm{x}$ & $\mathrm{y}$ & $\mathrm{z}$ & $\mathrm{a}$ & $\mathrm{b}$ & $\mathrm{c}$ \\
\hline Laser source & & $\delta y_{s}$ & $\delta z_{s}$ & & $\delta b_{s}$ & $\delta c_{s}$ \\
Rotary axis x & & $\delta y_{a x}$ & $\delta z_{a x}$ & $\delta a_{a x}$ & $\delta b_{a x}$ & $\delta c_{a x}$ \\
Mirror x & & & $\delta z_{m x}$ & $\delta a_{m x}$ & $\delta b_{m x}$ & \\
Rotary axis y & & $\delta y_{a y}$ & $\delta z_{a y}$ & $\delta a_{a y}$ & $\delta b_{a y}$ & $\delta c_{a y}$ \\
Mirror y & & & $\delta z_{m y}$ & $\delta a_{m y}$ & $\delta b_{m y}$ & \\
Window 1 & & & & $\delta a_{w 1}$ & $\delta b_{w 1}$ & \\
Window 2 & & & & $\delta a_{w 2}$ & $\delta b_{w 2}$ & \\
Work plane & $\delta x_{p}$ & $\delta y_{p}$ & $\delta z_{p}$ & $\delta a_{p}$ & $\delta b_{p}$ & $\delta c_{p}$ \\
\hline
\end{tabular}

- ${ }^{\text {mirror_x_d }} \boldsymbol{T}_{\text {mirror_x }}=\left(0,0, \delta z_{m x}, \delta a_{m x}, \delta b_{m x}, 0\right)$

- ${ }^{a x i s \_y 0 \_d} \boldsymbol{T}_{a x i s_{-} y 0}=\left(0, \delta y_{a y}, \delta z_{a y}, \delta a_{a y}, \delta b_{a y}, \delta c_{a y}\right)$

- ${ }^{\text {mirror_-y_d }} \boldsymbol{T}_{\text {mirror_y }}=\left(0,0, \delta z_{m y}, \delta a_{m y}, \delta b_{m y}, 0\right)$

- ${ }^{\text {window_1_d }} \boldsymbol{T}_{\text {window_1 }}=\left(0,0,0, \delta a_{w 1}, \delta b_{w 1}, 0\right)$

- ${ }^{\text {window_2_d }} \boldsymbol{T}_{\text {window_2 }}=\left(0,0,0, \delta a_{w 2}, \delta b_{w 2}, 0\right)$

- ${ }^{\text {plane_d }} \boldsymbol{T}_{\text {plane }}=\left(\delta x_{p}, \delta y_{p}, \delta z_{p}, \delta a_{p}, \delta b_{p}, \delta c_{p}\right)$

No simplifying assumptions are made on the transformation matrices, hence the $\delta_{i}$ defects are considered as new geometric parameters. Fig. 4 represents the system with defects.

The FKM with defects is built as before by following the optical path. Only the part concerning the writing of all vectors and points in the same coordinate system is modified by the addition of the new transformation matrices. Finally, the geometrical model obtained is composed of 46 parameters (30 assembly defects, 14 geometrical parameters and 2 optical parameters). Its mathematical expression is represented in Eq. (15) by the function $f(\boldsymbol{\delta}, \boldsymbol{Q})$ where $\delta$ represents the vector containing the 30 assembly defects $\delta=\left(\delta y_{s}, \ldots, \delta c_{p}\right)$.

$$
\boldsymbol{X}=f(\boldsymbol{\delta}, \boldsymbol{Q})=h\left(\delta y_{s}, \ldots, \delta c_{p}, \theta_{x}, \theta_{y}\right)
$$

If $\delta=(0, \ldots, 0)$ the FKM with defects is equal to the nominal FKM then $f(\boldsymbol{\delta}, \boldsymbol{Q})=h(\boldsymbol{Q})$. It is possible to linearize the model with defects with respect to the defects. However, the resulting model does not allow to achieve the desired accuracy.

As previously mentioned with the nominal model, the IKM with defects is solved numerically with a Newton-Raphson convergence algorithm. The forward and inverse kinematic models with defects allow to simulate the behavior of an additive manufacturing machine subjected to assembly defects. These models

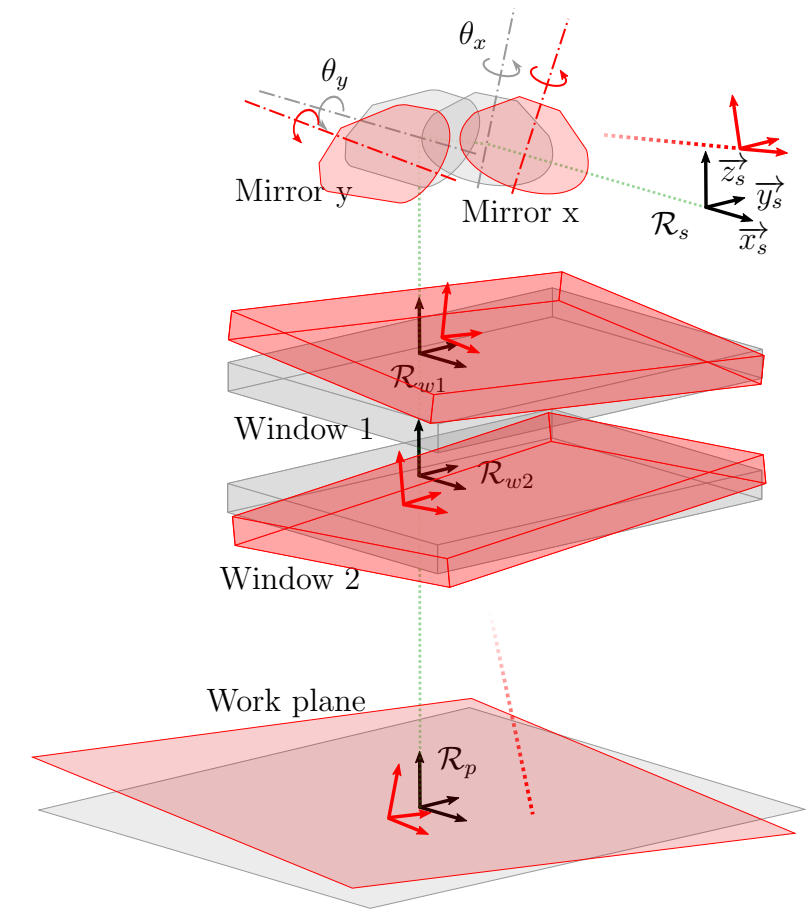

Figure 4: Optical chain parameterization with 30 assembly defects.

become expensive in computation time and thus cannot be used in real time in numerical control. Consequently, it is necessary to discretize these geometrical models in correction tables in order to be able to establish the joint commands in real time during optical chain control.

\subsection{Assembly defects influence}

By using the previous forward kinematic model, it is possible to quantify the influence of each assembly defect on the laser spot position. This helps to understand which defect have the highest impact on the behaviour of the optical chain.

Let be any virtual machine with defects $f(\boldsymbol{\delta}, \boldsymbol{Q})$ and a vector $\delta_{i}=\left(0, \ldots, 0, \delta_{i}, 0, \ldots, 0\right)$ representing the studied defect. The influence $I$ of a defect $\delta_{i}$ on this virtual machine corresponds to the difference between the laser spot position described by this virtual machine with the defect $\delta_{i}$ and the one without the defect $\delta_{i}$ (Eq. (16)).

$$
I\left(\boldsymbol{\delta}+\boldsymbol{\delta}_{i}, \boldsymbol{Q}\right)=f\left(\boldsymbol{\delta}+\boldsymbol{\delta}_{i}, \boldsymbol{Q}\right)-f(\boldsymbol{\delta}, \boldsymbol{Q})
$$

If $\delta=(0, \ldots, 0)$ then $I\left(\delta+\delta_{i}, Q\right)$ represents the influence of the defect $\delta_{i}$ on the nominal model. In the following, the influence 
of a defect is qualified as unitary when the value of the defect increment $\delta_{i}$ is equal to $1 \mathrm{~mm}$ for a position defect, or $1 \mathrm{mrad}$ for an orientation defect.

Fig. 5 shows the unitary influence of an orientation defect of the laser source around $\overrightarrow{z_{s}}\left(\delta c_{s}\right)$. This defect causes a nonuniform displacement of the laser spot position in the work plane. Deviation is minimal at the center of the work area and increases as it moves away from the center by following an ellipse. The displacement field follows the same direction, which depends on the mirror $x$ rotary axis orientation (parameter $B_{a x 0}$ ). A similar analysis can be performed for each of the 29 remaining defects. This analysis shows that the defects influence and their variation are often minimal in the center. For this reason, galvanometric systems are often used only on a limited portion of the reachable area. This allows to calibrate easily the scanning head using only polynomial approximations.

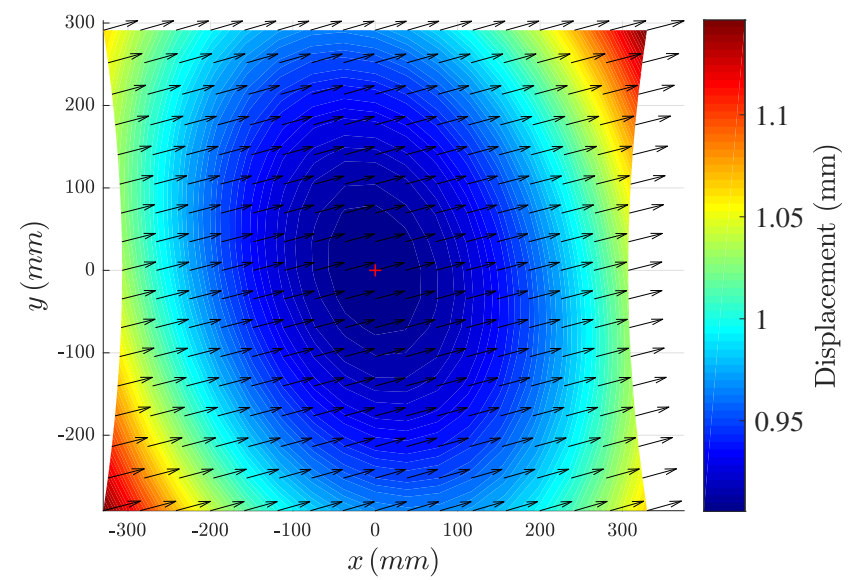

Figure 5: Influence on the nominal model of a unitary orientation defect of the laser source around $\overrightarrow{z_{s}}\left(\delta c_{s}=1 \mathrm{mrad}\right)$.

The comparison of the assembly defects influence on the nominal model is carried out using the maximum displacement criterion of the laser spot position in the work plane. The value of this displacement is shown in Fig. 6 for unitary orientation defects and in Fig. 7 for unitary position defects. The color indicates to which elements the defects are attached (gray for mirrors and axes, red for the laser, blue for the work plane, and yellow for the windows).

Fig. 6 allows to highlight several information. First of all, the four window orientation defects have a negligible influence on the laser spot position (1000 times less important than the other defects). The defects with the most impact come from the mirrors and their rotary axes. Laser source orientation defects have three times more influence on the laser spot position than work plane orientation defects. The study of Fig. 7 allows similar conclusions to be drawn. The magnitude of position defects is of the same order of magnitude as for orientation defects. Defects related to mirrors and their rotary axes are also predominant. However, for work plane defects, the influence of positioning defects is greater than the influence of orientation defects. All these results depend on the system geometry, but especially on the angular strokes of the actuators, which define the laser spot accessible area size on the work plane. The work carried out allows assembly defects to be classified by importance.

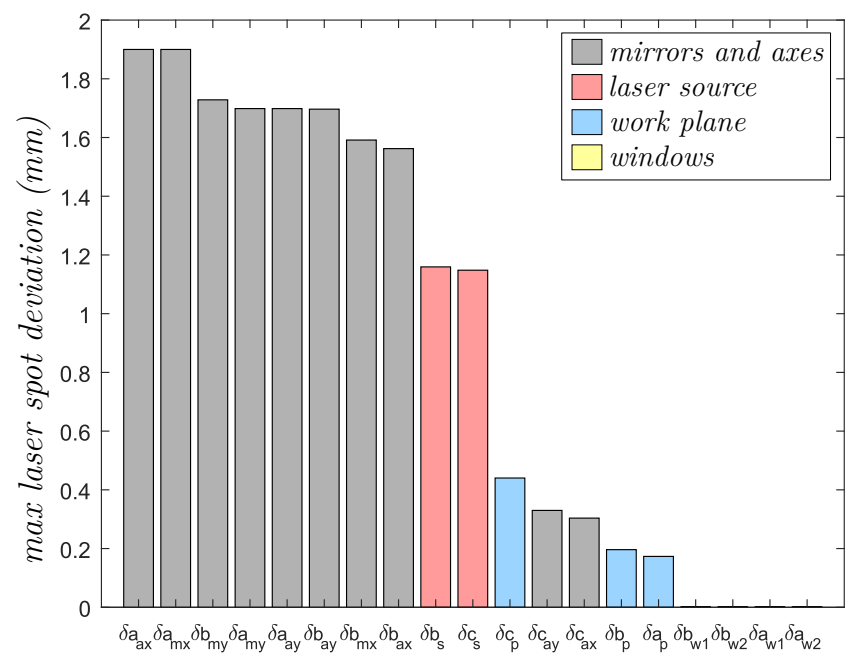

Figure 6: Influence of all unitary orientation defects on the nominal model.

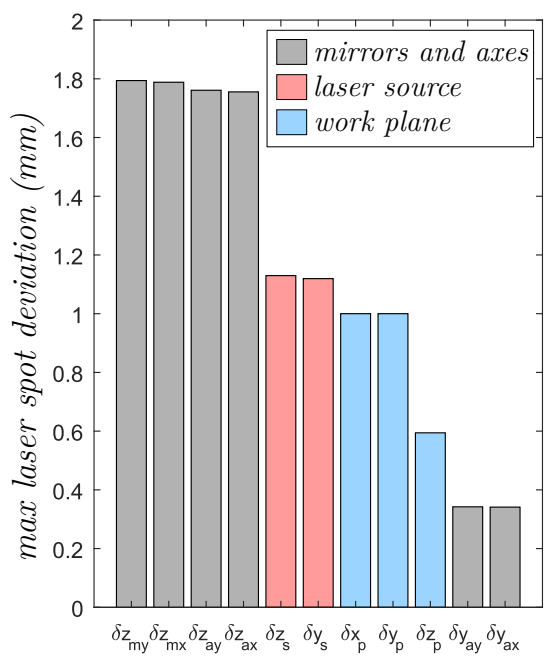

Figure 7: Influence of all unitary position defects on the nominal model.

\section{Assembly defect identification}

The geometrical model with defects developed in the previous section is used here to identify a virtual machine whose behavior tends towards the real system. To achieve this goal, a defect identification algorithm is implemented to readjust the model with defects on a set of experimental data.

\subsection{Assembly defect identification algorithm}

The identification of a virtual machine with defects reduces the calibration time of additive manufacturing machines. Indeed, the model can be readjusted to the real system by performing only one set of measurements. It is then sufficient to discretize the identified model to obtain the final correction tables required to operate the additive manufacturing machine. 


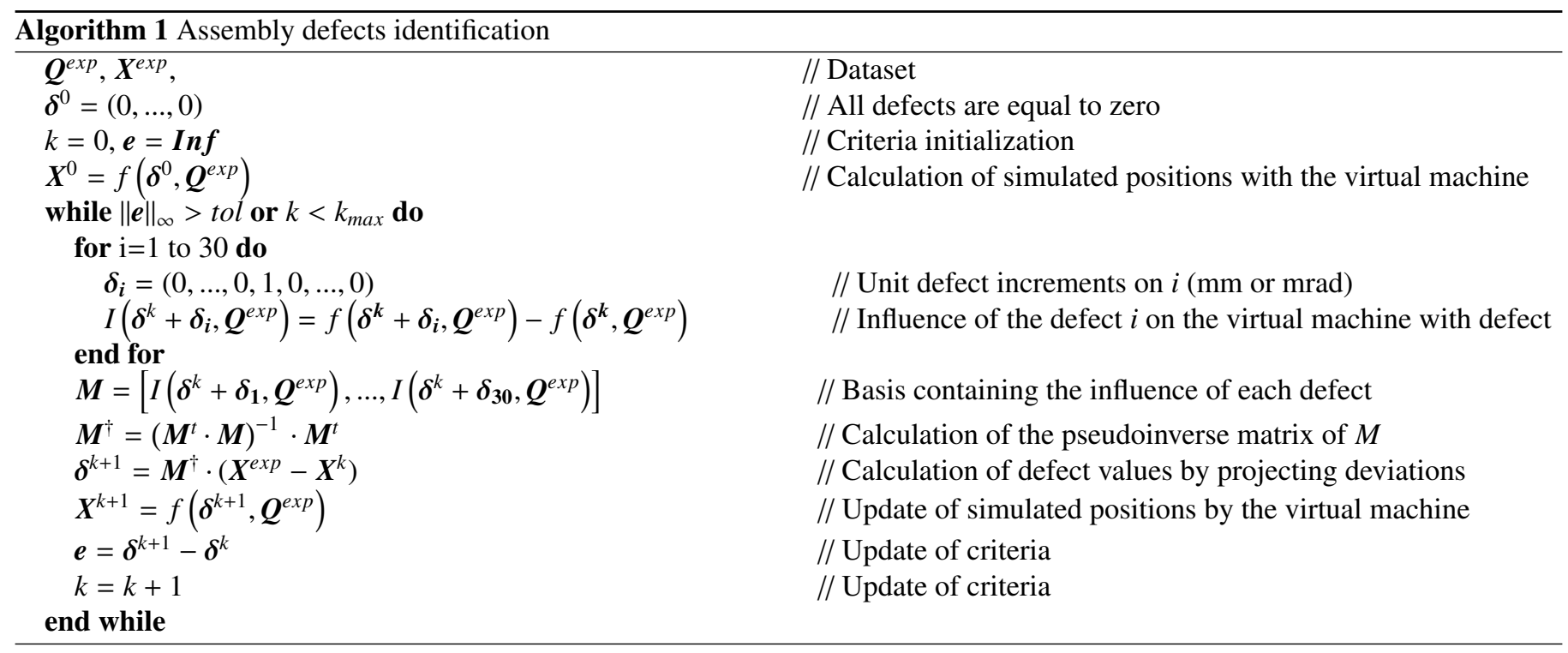

This calibration approach allows the system geometry to be taken into account when generating correction tables.

In order to identify a virtual machine, the algorithm relies on a dataset composed of different joint configurations $\boldsymbol{Q}^{\text {exp }}$ and corresponding positions in the task space $\boldsymbol{X}^{\exp }$. This dataset is generated without using a correction table in the numerical control and its dimension must be at least equal or greater than the number of defects to be identified. This dataset is used to identify a defect vector $\delta$ in order to readjust the geometrical model to the dataset. This approach is detailed in the algorithm 1 .

Initially, the machine is assumed to be perfect and corresponds to the nominal model, therefore the vector containing all defects is null $\delta^{0}=(0, \ldots, 0)$. Then in order to identify the contribution of each defect $\delta_{i}$ in the data $\boldsymbol{X}^{\exp }$, the influence of each defect $I\left(\delta^{0}+\delta_{i}, Q^{\text {exp }}\right)$ in each of the joint configurations $\boldsymbol{Q}^{\exp }$ is computed. The concatenation of all the defect influences generates a defect basis $\boldsymbol{M}$ depending on the machine configuration $\delta^{0}$ (Eq. (17)).

$$
\boldsymbol{M}=\left[I\left(\boldsymbol{\delta}^{0}+\boldsymbol{\delta}_{\mathbf{1}}, \boldsymbol{Q}^{\exp }\right), \ldots, I\left(\boldsymbol{\delta}^{0}+\boldsymbol{\delta}_{\mathbf{3 0}}, \boldsymbol{Q}^{\exp }\right)\right]
$$

This basis is then inverted to identify the contributions of each defect. Considering that the matrix $\boldsymbol{M}$ is not necessarily square, a pseudoinverse is performed (Eq. (18)).

$$
\boldsymbol{M}^{\dagger}=\left(\boldsymbol{M}^{t} \cdot \boldsymbol{M}\right)^{-1} \cdot \boldsymbol{M}^{t}
$$

Finally, the differences between the measured positions of the laser spot $\boldsymbol{X}^{\exp }$ and the simulated positions by the virtual machine $\boldsymbol{X}^{0}=f\left(\boldsymbol{\delta}^{0}, \boldsymbol{Q}^{\text {exp }}\right)$ are projected on the basis $\boldsymbol{M}^{\dagger}$ (Eq. (19)).

$$
\boldsymbol{\delta}^{1}=\boldsymbol{M}^{\dagger} \cdot\left(\boldsymbol{X}^{\exp }-\boldsymbol{X}^{0}\right)
$$

The defect vector $\boldsymbol{\delta}^{1}=\left(\delta^{1} y_{s}, \ldots, \delta^{1} c_{p}\right)$ is thus obtained. However, considering that the geometric model with defect is nonlinear with respect to defects, it is necessary to iterate the previous algorithm to converge to the final associated virtual machine. To do this, the defect basis is recalculated by considering the previously determined virtual machine $f\left(\boldsymbol{\delta}^{1}, \boldsymbol{Q}^{\exp }\right)$.
The iteration stops if the variation of all the identified defects are small enough $(t o l)$ or if the number of iterations reaches a threshold value $\left(k_{\max }\right)$.

\subsection{Results from a virtual machine}

First, to test the efficiency of the identification algorithm without influence of noise from the measurement (of $\boldsymbol{X}^{\exp }$ on the real machine), a dataset $\boldsymbol{Q}^{\exp }, \boldsymbol{X}^{\exp }$ is generated using a reference virtual machine with known defects. The objective is then to compare the identified parameters through the algorithm with the ones of the reference virtual machine. This dataset is introduced into the identification algorithm in order to find the corresponding parameters of the virtual machine. Fig. 8 shows the deviations of the laser spot position between the reference virtual machine and the identified virtual machine. The distribution of deviations is not homogeneous and the maximum deviation $\left(16 \cdot 10^{-6} \mathrm{~mm}\right)$ is located in the corners of the work plane. This test shows the relevance of the proposed algorithm.

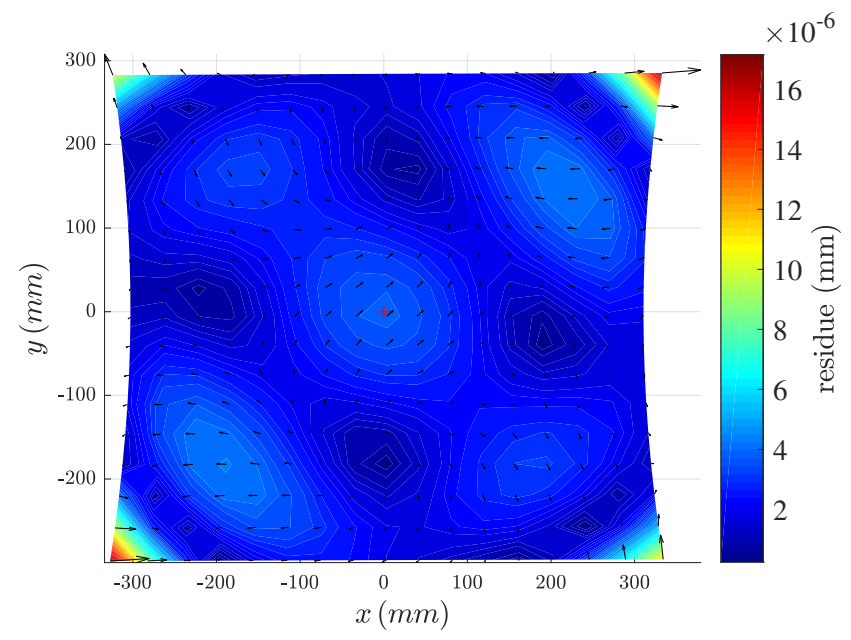

Figure 8: Residue between the initial data and the identified virtual machine. 
Table 2: Values of the imposed assembly defects, and of the identified assembly defects. Results are expressed in mm or mrad.

\begin{tabular}{|c|c|c|c|c|c|c|c|c|c|c|c|c|c|c|c|c|}
\hline \multicolumn{3}{|c|}{ defect (mm or mrad) } & $\delta y_{s}$ & $\delta z_{s}$ & $\delta y_{a x}$ & $\delta z_{a x}$ & $\delta z_{m x}$ & $\delta y_{a y}$ & $\delta z_{a y}$ & $\delta z_{m y}$ & $\delta x_{p}$ & $\delta y_{p}$ & $\delta z_{p}$ & $\delta b_{s}$ & $\delta c_{s}$ & \\
\hline \multirow{2}{*}{\multicolumn{3}{|c|}{$\begin{array}{l}\text { reference parameters } \\
\text { identified parameters }\end{array}$}} & 0.8 & 1.8 & -1 & -2 & -0.5 & 1 & 1.9 & -1.4 & 1.6 & -1.1 & 1 & -0.5 & 1.3 & \\
\hline & & & 0.15 & 1.59 & 0.14 & -0.23 & -0.5 & 1.61 & 1.73 & -1.40 & -0.06 & 0.19 & 0.83 & -0.10 & 0.28 & \\
\hline$\delta a_{a x}$ & $\delta b_{a x}$ & $\delta c_{a x}$ & $\delta a_{m x}$ & $\delta b_{m x}$ & $\delta a_{a y}$ & $\delta b_{a y}$ & $\delta c_{a y}$ & $\delta a_{m y}$ & $\delta b_{m y}$ & $\delta a_{w 1}$ & $\delta b_{w 1}$ & $\delta a_{w 2}$ & $\delta b_{w 2}$ & $\delta a_{p}$ & $\delta b_{p}$ & $\delta c_{p}$ \\
\hline-0.3 & -1.7 & 1.2 & 0.5 & -2 & 0.8 & -0.9 & 0.5 & -0.2 & 0.5 & 0.2 & 1.6 & 0.7 & -0.8 & -1.2 & 0.6 & 0.7 \\
\hline 0.42 & -1.04 & 0.93 & 0.42 & -2.0 & 0.09 & -0.98 & 1.26 & 0.09 & 0.5 & 0 & 0 & 0 & 0 & -0.10 & 0.07 & 0.14 \\
\hline
\end{tabular}

Table 2 shows that the values of the identified defects are different from the simulation values. This difference can be explained mathematically because the basis composed of the 30 defects is not orthogonal and multiple solutions of the inversion of $M$ can be found, therefore two different machine configurations with defects can generate the same laser spot positioning defect.

To validate the robustness of the identification algorithm 1 another test is performed. A dataset $\boldsymbol{Q}^{\exp }, \boldsymbol{X}^{\exp }$ has been generated using a virtual machine and a uniformly distributed random noise has been added to each component $x$ and $y$ of the dataset $\boldsymbol{X}^{\text {exp }}$ in order to represent all possible defects influence which are not considered in this study (measurement uncertainties of marked plates, thermal defects of the opto-mechanical chain ...). The noisy dataset is set between $\pm 0.05 \mathrm{~mm}$ and used to identify a virtual machine.

The results lead to the following conclusion: the maximal deviation between the identified virtual machine and the noisy data $\boldsymbol{X}^{\exp }$ is equal to $0.0705 \mathrm{~mm}$. This value is coherent because it also represents the maximal possible deviation introduces by the noise $(0.05 \cdot \sqrt{2})$. The mean deviation is $0.038 \mathrm{~mm}$ and the standard deviation is $0.014 \mathrm{~mm}$. But the most interesting part is to compare the data without noise $\boldsymbol{X}^{\text {exp }}$ and the identified virtual machine. In this case, the maximal deviation is equal to $0.007 \mathrm{~mm}$, the mean of the deviations is $0.022 \mathrm{~mm}$ and the standard deviation is $0.004 \mathrm{~mm}$. The proposed algorithm is therefore relatively not very sensitive to noise and allows to identify a virtual machine by performing an overall minimization of deviations.

The behaviour of the virtual machine with defects identified thanks to the proposed algorithm tends towards the real machine. The convergence of this algorithm is fast. In the previous example, after 3 iterations the variation of the laser spot position on work plane between two consecutive iterations is less than $1 \mu \mathrm{m}$.

\subsection{Results based on data from a real machine}

The virtual machine identification procedure was used with real data coming from an industrial LBM machine. To obtain the dataset $\boldsymbol{Q}^{\text {exp }}, \boldsymbol{X}^{\text {exp }}$, the machine is preheated in order to consider the behaviours related to the system dilatation and to the thermal deformations of mirrors. Then a grid is engraved on a reference plate using the additive manufacturing machine. The measurement of the distance between the intersections of the grid and the reference already present is performed using a measuring system. Hence, the positions in the task space $\boldsymbol{X}^{\exp }$ are extracted with a $10 \mu \mathrm{m}$ uncertainty. The corresponding joint positions $\boldsymbol{Q}^{\text {exp }}$ are obtained by monitoring the encoders feedback. The measurement uncertainty is here $2.3 \cdot 10^{-8} \mathrm{rad}$. The resulting dataset therefore includes all possible defects of the opto-mechanical chain (mechanical, thermal, optical defects, etc.) as well as defects due to the measurement of this data set (sensor resolution, noise, uncertainty, etc.). All these defects are not modeled in our approach, therefore, the 30 assembly defects identified do not represent only real assembly defects, but rather parameters that also include other effects and so minimize the deviations between the virtual machine and the real machine.

Fig. 9 shows the differences between the nominal model (without defect) and the actual data. These differences are between 0 and $1.2 \mathrm{~mm}$ for a work plane of $350 \mathrm{mmm} \times 540 \mathrm{~mm}$. For this same working zone, the models proposed in the literature [2], [3], [8] have maximum deviations of $2.3 \mathrm{~mm}$. The imprecision of the literature models is largely due to the fact that the point named $P_{2}$ is located on the axis of rotation of the mirror $y$ whatever the configuration $\left(\theta_{x}, \theta_{y}\right)$. However, it appears that this point moves on the mirror surface $y$ considering the distance $e_{m}$ between the mirrors and their rotation axes.

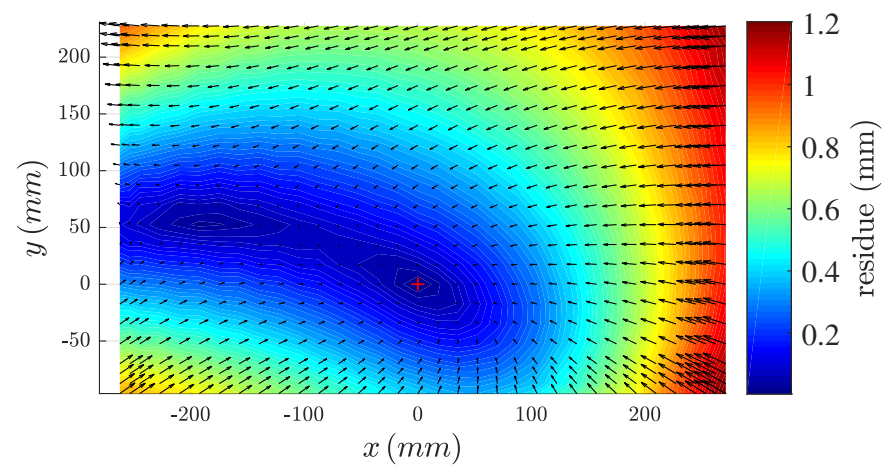

Figure 9: Residue between measurements and model without defect (nominal model).

In order to obtain a model closer to the real system, a virtual machine with defect is identified using the protocol previously presented. Fig. 10 shows the deviations between the identified virtual machine and the actual marked grid. Fig. 11 displays the cumulative histogram of the deviations distribution. Re- 
sults show that $60 \%$ of the measured deviations are less than $10 \mu \mathrm{m}$ and $95 \%$ are less than $20 \mu \mathrm{m}$. This calibration method minimizes the error between the actual data and the simulated data as the whole. However, some local errors cannot be compensated. On Fig. 10, a local defect of $50 \mu \mathrm{m}$ is observed on the lower right corner. This error may be due to a local measurement error or another local source of defect not taken into account in our defects basis (imperfection on the windows surfaces or mirror surfaces, non-linearity of the control system, local deformation of the mirror reflection surface under thermal stress, modification of the shape of the laser spot on the work area extremities and therefore of the engraving trace ...). In this case, the machine working area is smaller than the calibrated area. This eliminates the impacts of defects often present on the borders of the calibrated area and allows a maximum error below $25 \mu \mathrm{m}$ on the entire working area of the machine. The efficiency of the method has been validated on a LPBF machine with industrial environment. Calibration process is performed in a single shot on the plate, reducing the time required for adjustment and installation of the machine.

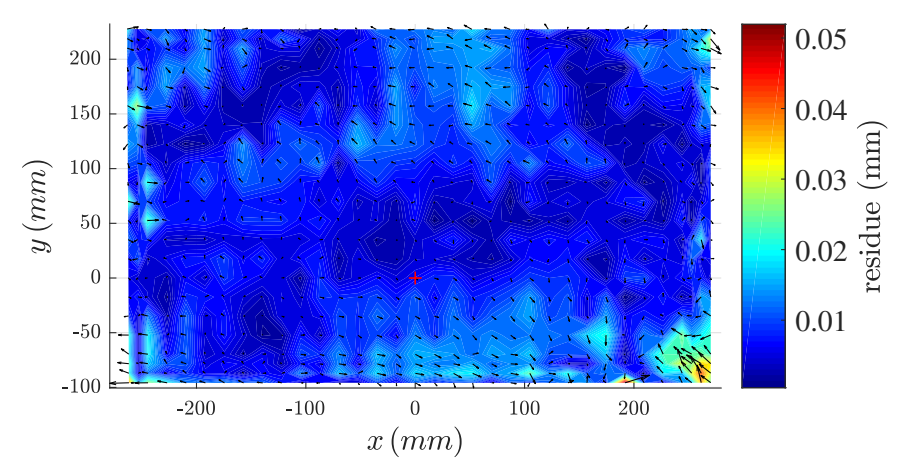

Figure 10: Residue between the measurements and the identified model.

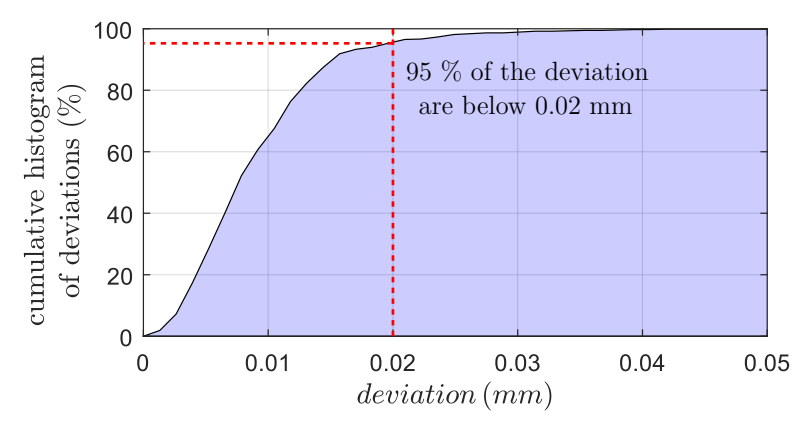

Figure 11: Cumulative histogram of deviations from Fig. 10 (in \%).

\section{Conclusion}

This paper presents two mathematical models developed to simulate the behaviour of the opto-mechanical chain of an additive manufacturing machine: a nominal model and a model considering assembly defects of each component of the scanhead. These models allow to analyse and to quantify the impact of each assembly defect on the laser spot position in the work plane. Moreover these models show that assembly defects have less influence at the center of the work area than at the extremities. Based on these models, parameters identification is proposed to fit the virtual machine including defects to the real machine. This virtual machine is used for the calibration of the system and for generating correction tables. Tests performed on an industrial machine proved the efficiency of the method. $95 \%$ of deviations of the laser spot position are less than $20 \mu \mathrm{m}$ on the calibrated area and $100 \%$ are less than $25 \mu \mathrm{m}$ in the working area. The proposed approach ensures significant time savings during calibration taking advantage of a single real measurement phase. The work developed in this article can be easily transposed to post-objective scanning systems (f-theta lens).

\section{References}

[1] J. S. Ehrmann, Optics for vector scanning, Proc. SPIE 1454 (1991) 245256. doi:10.1117/12.28036.

[2] A. Nee, J. Fuh, T. Miyazawa, On the improvement of the stereolithography (sl) process, Journal of Materials Processing Technology 113 (1-3) (2001) 262 - 268. doi:10.1016/s0924-0136(01)00634-3.

[3] J. Xie, S. Huang, Z. Duan, Y. Shi, S. Wen, Correction of the image distortion for laser galvanometric scanning system, Optics \& Laser Technology 37 (4) (2005) 305 - 311. doi:10.1016/j.optlastec.2004.04.012.

[4] M. A. O. Delgado, A. F. Lasagni, Reducing field distortion for galvanometer scanning system using a vision system, Optics and Lasers in Engineering 86 (2016) 106 - 114. doi:10.1016/j.optlaseng.2016.05.016.

[5] P. E. Verboven, Distortion correction formulas for pre-objective dual galvanometer laser scanning, Appl. Opt. 27 (20) (1988) 4172-4173. doi:10.1364/AO.27.004172.

[6] Software calibration of scan system distortions, Vol. 1454. doi: $10.1117 / 12.28040$.

[7] M.-F. Chen, Y.-P. Chen, Compensating technique of field-distorting error for the co2 laser galvanometric scanning drilling machines, International Journal of Machine Tools and Manufacture 47 (7) (2007) 1114 - 1124. doi:10.1016/j.ijmachtools.2006.09.015.

[8] A. Manakov, H.-P. Seidel, I. Ihrke, A mathematical model and calibration procedure for galvanometric laser scanning systems., in: P. Eisert, J. Hornegger, K. Polthier (Eds.), VMV, Eurographics Association, 2011, pp. 207-214.

[9] P. Brosens, Scanning accuracy of the moving-iron galvanometer scanner, Optical Engineering 15 (2) (1976) 295. doi:10.1117/12.7971926.

[10] J. R. D. P. Jablonowski, Beam deflection at high accuracy and precision (1977). doi:10.1117/12.954921.

[11] F. Blais, Control of low inertia galvanometers for high precision laser scanning systems, Optical Engineering 27 (2) (1988) 104. doi:10.1117/12.7976653.

[12] P. J. Brosens, Dynamic mirror distortions in optical scanning, Applied Optics 11 (12) (1972) 2987-2989. doi:10.1364/AO.11.002987.

[13] K. Seki, H. Kannami, M. Iwasaki, H. Hirai, Application of selfsensing actuation using piezoelectric element for vibration suppression of galvanometric mirror, in: 2010 IEEE/ASME International Conference on Advanced Intelligent Mechatronics, 2010, pp. 1070-1075. doi:10.1109/AIM.2010.5695756.

[14] D. Matsuka, T. Tanaka, M. Iwasaki, Thermal demagnetization compensation for fast and precise positioning in galvanometer scanners, IEEE Transactions on Industrial Electronics 63 (9) (2016) 5514-5522. doi:10.1109/TIE.2016.2561878.

[15] G. Eberle, C. Dold, K. Wegener, Building a vector model representation of a two-axis laser scanhead using numerical analysis for simulation purposes, IJMIC 20 (2013) 199-207. doi:10.1504/IJMIC.2013.057131.

[16] J. R. R. Mayer, G. A. Parker, A portable instrument for 3-d dynamic robot measurements using triangulation and laser tracking, IEEE Transactions on Robotics and Automation 10 (4) (1994) 504-516. doi: $10.1109 / 70.313100$. 